\title{
Release mechanism of high mobility group nucleosome binding domain 1 from lipopolysaccharide-stimulated macrophages
}

\author{
TAISUKE MURAKAMI $^{1}$, ZHONGSHUANG HU ${ }^{1}$, HIROSHI TAMURA ${ }^{2}$ and ISAO NAGAOKA ${ }^{1}$ \\ ${ }^{1}$ Department of Host Defense and Biochemical Research, Juntendo University, Graduate School of Medicine, \\ Tokyo 113-8421; ${ }^{2}$ Laboratory Program Support Consulting Office, Tokyo 160-0023, Japan
}

Received March 3, 2015; Accepted December 15, 2015

DOI: $10.3892 / \mathrm{mmr} .2016 .4893$

\begin{abstract}
Alarmins are identified as endogenous mediators that have potent immune-activating abilities. High mobility group nucleosome binding domain 1 (HMGN1), a highly conserved, non-histone chromosomal protein, which binds to the inner side of the nucleosomal DNA, regulates chromatin dynamics and transcription in cells. Furthermore, HMGN1 acts as a cytokine in the extracellular milieu by inducing the recruitment and maturation of antigen-presenting cells (dendritic cells) to enhance Th1-type antigen-specific immune responses. Thus, HMGN1 is expected to act as an alarmin, when released into the extracellular milieu. The present study investigated the release mechanism of HMGN1 from macrophages using mouse macrophage-like RAW264.7 cells. The results indicated that HMGN1 was released from lipopolysaccharide (LPS)-stimulated RAW264.7 cells, accompanied by cell death as assessed by the release of lactate dehydrogenase (LDH). Subsequently, the patterns of cell death involved in HMGN1 release from LPS-stimulated RAW264.7 cells were determined using a caspase-1 inhibitor, YVAD, and a necroptosis inhibitor, Nec-1. YVAD and Nec-1 did not alter LPS-induced HMGN1 and LDH release, suggesting that pyroptosis (caspase-1-activated cell death) and necroptosis are not involved in the release of HMGN1 from LPS-stimulated RAW264.7 cells. In addition, flow cytometric analysis indicated that LPS stimulation did not induce apoptosis but substantially augmented necrosis, as evidenced by staining with annexin V/propidium iodide. Together these
\end{abstract}

Correspondence to: Professor Isao Nagaoka, Department of Host Defense and Biochemical Research, Juntendo University, Graduate School of Medicine, 2-1-1 Hongo, Bunkyo-ku, Tokyo 113-8421, Japan

E-mail: nagaokai@juntendo.ac.jp

Abbreviations: HMGN1, high mobility group nucleosome binding domain 1; LPS, lipopolysaccharide; HMGB1, high mobility group box 1; HMG, high mobility group; NF- $\kappa \mathrm{B}$, nuclear factor $\kappa \mathrm{B}$; MAPK, mitogen activated protein kinase

Key words: alarmin, endotoxin shock, sepsis, high mobility group nucleosome binding domain 1, macrophage findings suggest that HMGN1 is extracellularly released from LPS-stimulated RAW264.7 macrophage-like cells, accompanied by unprogrammed necrotic cell death but not pyroptosis, necroptosis or apoptosis.

\section{Introduction}

Sepsis is caused by polymicrobial infections associated with severe systemic inflammatory response syndrome that leads to multiple organ failure, including acute lung injury and renal and hepatic failure, as well as septic shock (1-3). Bacterial endotoxin lipopolysaccharide (LPS) is a major component of the outer membrane of gram-negative bacteria and is important for stimulating mononuclear phagocytes (macrophages and monocytes) to secrete various inflammatory mediators (e.g. cytokines, reactive oxygen species, prostanoids/leukotrienes, proteases and nitric oxide), including alarmins $(1,3,4)$.

Alarmins are identified as endogenous mediators that have potent immune-activating abilities. To date, distinct molecules of alarmins have been identified, including antibacterial peptide (defensin and cathelicidin), cytokines (IL-1 $\beta$ and IL-33), heat shock proteins, adenosine triphosphate, uric acid and nuclear components (HMG proteins, nucleosome and histone) (4-9). Generally, alarmins are extracellularly released by the following mechanisms: i) Passive release by necrotic (unprogrammed) cell death, which is caused by infective or noninfective tissue damage or ii) active release by certain immune cells without cell death by a specialized secretion pathway, in which alarmins are transported from the nucleus to the cytoplasm, then into the lysosome and eventually released into the extracellular space through exocytosis $(9,10)$. Additionally, previous studies have revealed that pyroptosis, a form of programmed necrotic cell death accompanied by the formation of inflammasomes, caspase- 1 activation and IL- $1 \beta$ production, induces the secretion of certain alarmins $(11,12)$. Alarmins augment the innate and adaptive immune responses by distinct mechanisms and are capable of promoting the recruitment and activation of immune cells, including antigen-presenting cells $(13,14)$. Under septic conditions, excessive release of several alarmins, including HMGB1 and IL-1 $\beta$, leads to uncontrolled inflammation in the host $(15,16)$. By contrast, alarmins also exhibit beneficial effects on the host by promoting the mobilization and activation of immune cells to eliminate 
potential pathogens $(13,14)$. Thus, alarmins are crucial in the pathological process of sepsis/septic shock.

High mobility group nucleosomal binding protein-1 (HMGN1), a highly conserved, non-histone chromosomal protein, which binds to the inner side of the nucleosomal DNA, regulates chromatin dynamics and transcription in cells $(17,18)$. By contrast, HMGN1 acts as a cytokine in the extracellular milieu. HMGN1 induces the recruitment and maturation of antigen-presenting cells (dendritic cells) to enhance Th1-type antigen-specific immune responses (13). In addition, HMGN1 promotes the secretion of inflammatory cytokines from dendritic cells via direct interaction with Toll-like receptor 4 (TLR4), followed by the activation of myeloid differentiation primary response gene 88 /nuclear factor $\kappa \mathrm{B}(\mathrm{NF}-\kappa \mathrm{B})$ and the mitogen activated protein kinase (MAPK) pathway (14). Thus, HMGN1 is expected to act as an alarmin, when released into the extracellular milieu, and to be important in the pathogenesis of sepsis/septic shock. However, the mechanism of HMGN1 release from immune cells remains to be elucidated. The present study therefore investigated the release mechanism of HMGN1 from macrophages, which is important in sepsis/septic shock by producing inflammatory mediators in response to bacterial pathogen-associated molecular patterns (PAMPs), using mouse macrophage-like RAW264.7 cells.

\section{Materials and methods}

Reagents. LPS (from E. coli serotype O111:B4) was purchased from Sigma-Aldrich (St. Louis, MO, USA). Ac-Tyr-Val-Ala-Asp-H (Ac-YVAD-CHO), a specific caspase-1 inhibitor was purchased from Peptide Institute, Inc. (Osaka, Japan). Necrostatin-1 (Nec-1), a selective inhibitor of necroptosis and its inactive analog necrostatin-1 (Nec-1i) were purchased from Merck Millipore (Darmstadt, Germany).

Quantification of HMGN1 released from LPS-stimulated RAW264.7 cells. A murine macrophage cell line RAW264.7 was purchased from the European Collection of Cell Cultures (Wiltshire, UK) and maintained in Dulbecco's modified Eagle's medium (DMEM; Sigma-Aldrich) supplemented with $10 \%$ fetal calf serum (endotoxin level <10 EU/ml; Cell Culture Technologies, Herndon, VA, USA) and 100 U/ml penicillin $/ 100 \mu \mathrm{g} / \mathrm{ml}$ streptomycin (Nacalai Tesque, Inc., Kyoto, Japan) at $37^{\circ} \mathrm{C}$ in a $5 \% \mathrm{CO}_{2}$ incubator. RAW264.7 cells $\left(2 \times 10^{5}\right.$ cells/well) were seeded into a 12 -well culture plate and incubated overnight at $37^{\circ} \mathrm{C}$ in $5 \% \mathrm{CO}_{2}$. Subsequently, cells were washed with fresh media and incubated with or without LPS $(100 \mathrm{ng} / \mathrm{ml})$ in the absence or presence of Ac-YVAD-CHO, $\mathrm{Nec}-1$ or Nec-1i at a final concentration of $10 \mu \mathrm{M}$ (each) in the media. Culture supernatants were recovered at $20 \mathrm{~h}$ after the incubation and HMGN1 in the culture supernatants was quantitated by western blotting. Prior to electrophoresis, supernatants were concentrated by trichloroacetic acid (TCA) precipitation. Briefly, $900 \mu \mathrm{l}$ of supernatants were added with ice-cold 1/9 volume of $100 \%$ TCA $(100 \mu \mathrm{l})$, vortexed and incubated on ice for $30 \mathrm{~min}$. The mixtures were centrifuged at $8,000 \times \mathrm{g}$ at $4^{\circ} \mathrm{C}$ for $15 \mathrm{~min}$ and the supernatants were aspirated. Pellets were washed with $1 \mathrm{ml}$ of $100 \%$ ice-cold acetone and centrifuged at $8,000 \mathrm{x} \mathrm{g}$ at $4^{\circ} \mathrm{C}$ for $5 \mathrm{~min}$, and dissolved in 1X Laemmli sample buffer. Dissolved samples were subjected to $15 \%$ sodium dodecyl sulfate-polyacrylamide gel electrophoresis (Nacalai Tesque, Inc.), and the resolved proteins were electrophoretically transferred onto polyvinylidine difluoride nitrocellulose membranes (Immobilon-P; Merck Millipore). The membranes were blocked in Blocking One (Nacalai Tesque, Inc.) and sequentially probed with rabbit anti-HMGN1 polyclonal antibody ( $2 \mu \mathrm{g} / \mathrm{ml}$; cat. no. ab5212; Abcam, Cambridge, MA, USA), and horseradish peroxidase-conjugated goat anti-rabbit IgG polyclonal antibody (1:2,000 dilution; cat. no. AP187P; Chemicon International, Temecula, CA, USA). HMGN1 was finally visualized using Super Signal West Dura Chemiluminescent Substrate (Pierce Biotechnology, Inc., Rockford, IL, USA) and the detected bands were analyzed using Multi Gauge software (version 3.0) and an LAS-3000 Image Analyzer (Fujifilm, Tokyo, Japan).

Quantification of lactate dehydrogenase (LDH) activities. LDH activity in the supernatants of LPS-stimulated RAW264.7 cells was determined for evaluating cell death. LDH activity in the supernatants and 1\% Triton X-100-lysed cells (as a total activity of $100 \%$ ) were measured using a commercially available LDH assay kit (Takara Bio, Inc., Shiga, Japan), according to the manufacturer's instructions.

Quantification of $I L-1 \beta$. The quantity of IL- $1 \beta$ in the culture supernatants of LPS-stimulated RAW264.7 cells was measured using a commercially available mouse IL-1 $\beta$ ELISA kit (detection limits of $8 \mathrm{pg} / \mathrm{ml}$; eBioscience, San Diego, CA, USA), according to the manufacturer's instructions.

Quantification of apoptosis. Apoptotic and necrotic cell death was assessed by staining cells with annexin V-fluorescein isothiocyanate and propidium iodide (PI). RAW264.7 cells $\left(2 \times 10^{5}\right.$ cells/well) were seeded into a 12 -well culture plate and incubated overnight at $37^{\circ} \mathrm{C}$ in $5 \% \mathrm{CO}_{2}$. Subsequently, cells were washed with fresh media and incubated with or without LPS $(100 \mathrm{ng} / \mathrm{ml})$ in the absence or presence of Ac-YVAD-CHO, Nec-1 or Nec-1i (10 $\mu \mathrm{M}$ each). After $8 \mathrm{~h}$, cells were washed with $0.05 \%$ ethylenediaminetetraacetic acid (EDTA)/phosphate-buffered saline (PBS) and detached with $0.25 \%$ trypsin-EDTA (Life Technologies; Thermo Fisher Scientific, Inc., Waltham, MA, USA). Subsequently, annexin V/PI staining was performed using a MEBCYTO Apoptosis kit (Medical \& Biological Laboratories Co., Ltd., Nagoya, Japan), according to the manufacturer's instructions. Briefly, cells were centrifuged in a $1.5 \mathrm{ml}$ tube $(200 \mathrm{x} \mathrm{g}$, $5 \mathrm{~min}$ ) and supernatants were discarded. Cells were sequentially rinsed with DMEM and ice-cold PBS, and resuspended in $85 \mathrm{ml}$ of binding buffer. A total of $10 \mu \mathrm{l}$ of annexin $\mathrm{V}$ stock solution and $5 \mu \mathrm{l}$ of PI stock solution was then added to the cells, and incubated for $15 \mathrm{~min}$ in the dark at room temperature. Thereafter, cells were immediately analyzed by flow cytometry (FACSCalibur cell analyzer; Becton-Dickinson, San Jose, CA, USA).

Statistical analysis. Data are presented as the mean \pm standard deviation. Statistical significance was determined by Student's t-test or one-way analysis of variance (GraphPad 
A

\section{Resting LPS +YVAD +Nec-1 +Nec-1i}

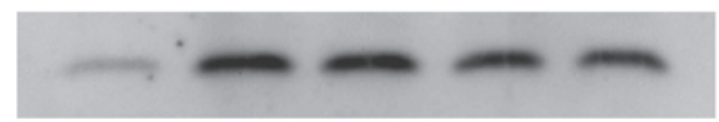

B

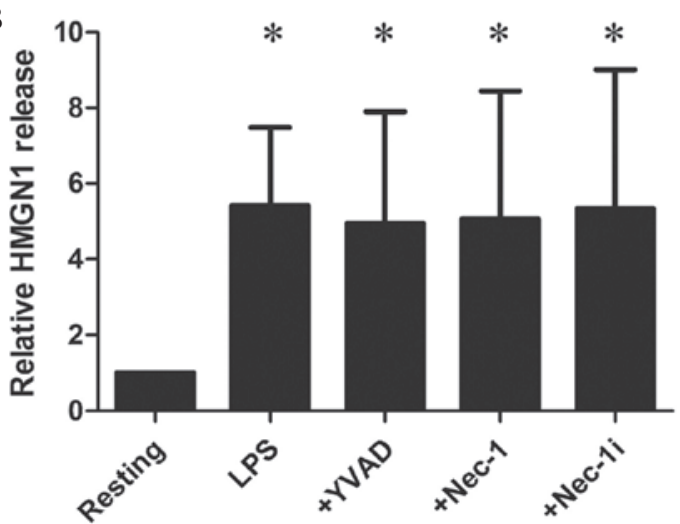

Figure 1. Release of HMGN1 from LPS-stimulated RAW264.7 cells (A) RAW264.7 cells were incubated with LPS $(100 \mathrm{ng} / \mathrm{ml})$ or without (resting) in the absence or presence of YVAD, Nec-1 or Nec-1i (10 $\mu \mathrm{M}$ each) at $37^{\circ} \mathrm{C}$ for $20 \mathrm{~h}$. HMGN1 was detected by western blotting. Images are representative of eight separate experiments. (B) HMGN1 release is expressed as a fold increase relative to resting. Data are presented as the mean \pm standard deviation of eight independent experiments. Values are compared between resting and LPS, +YVAD, $+\mathrm{Nec}-1$ or $+\mathrm{Nec}-1 \mathrm{i}$. ${ }^{*} \mathrm{P}<0.05$. HMGN1, high mobility group nucleosome binding domain 1; LPS, lipopolysaccharide; Nec-1, Necrostatin-1.

Prism Software Inc., La Jolla, CA, USA) with Tukey's post hoc test. $\mathrm{P}<0.05$ was considered to indicate a statistically significant difference.

\section{Results}

Release of HMGN1 from LPS-stimulated RAW264.7 cells. Initially, the present study aimed to determine whether LPS stimulation induces the extracellular release of HMGN1 from RAW264.7 cells. As shown in Fig. 1, the level of HMGN1 in the supernatants increased by 5.4-fold following LPS $(100 \mathrm{ng} / \mathrm{ml})$ stimulation compared with resting $(\mathrm{P}<0.05)$. The percentage of HMGN1 release was calculated from the total quantity of HMGN1 in the cell lysates. HMGN1 release was $<1 \%$ in resting cells; however, increased up to $2.7-5.9 \%$ in LPS-stimulated cells (data not shown).

Furthermore, LDH assay indicated that the LDH activity in the supernatants increased from $11.2 \pm 4.9 \%$ in resting cells to $21.5 \pm 6.0 \%$ in LPS-stimulated cells $(\mathrm{P}<0.05$; Fig. 2$)$, suggesting that HMGN1 release is accompanied by cell death.

Effects of Ac-YVAD-CHO and Nec-1 on HMGN1 release from LPS-stimulated RAW264.7 cells. Subsequently, the patterns of cell death involved in HMGN1 release from LPS-stimulated RAW264.7 cells were determined using Ac-YVAD-CHO (YVAD) and Nec-1. YVAD, a selective inhibitor of caspase 1, suppresses pyroptosis, whereas Nec-1, an allosteric inhibitor of the death domain receptor-associated

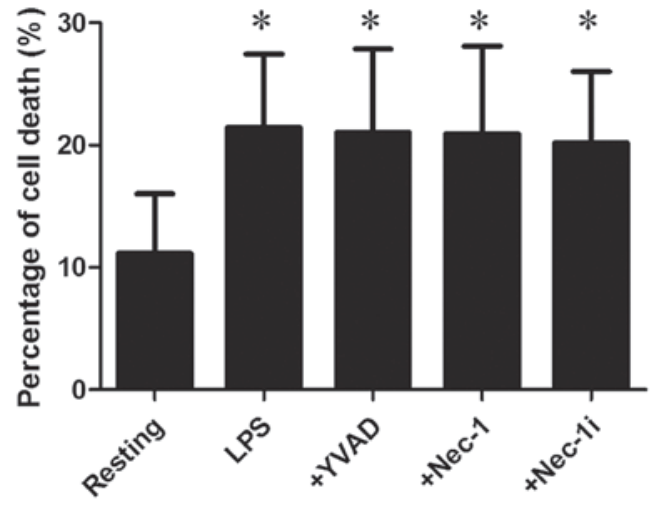

Figure 2. Release of LDH from LPS-stimulated RAW264.7 cells. RAW264.7 cells were incubated with LPS $(100 \mathrm{ng} / \mathrm{ml})$ or without (resting) in the absence or presence of YVAD, Nec-1 or Nec-1i $\left(10 \mu \mathrm{M}\right.$ each) at $37^{\circ} \mathrm{C}$ for $20 \mathrm{~h} . \mathrm{LDH}$ was quantitated using a commercially available LDH assay kit. Data are presented as the mean \pm standard deviation of 10 independent experiments. Values are compared between resting and LPS, +YVAD, $+\mathrm{Nec}-1$ or $+\mathrm{Nec}-1 \mathrm{i}$. "P $<0.05$. LPS, lipopolysaccharide; LDH, lactate dehydrogenase; Nec-1, Necrostatin-1.

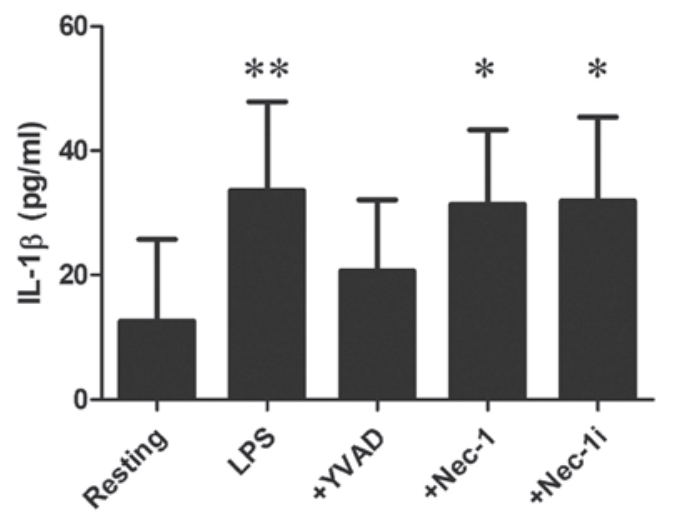

Figure 3. Release of IL-1 $\beta$ from LPS-stimulated RAW264.7 cells. RAW264.7 cells were incubated with LPS (100 ng/ml) or without (resting) in the absence or presence of YVAD, Nec-1 or Nec-1i (10 $\mu \mathrm{M}$ each) at $37^{\circ} \mathrm{C}$ for $20 \mathrm{~h}$, and IL-1 $\beta$ was quantitated by enzyme-linked immunosorbent assay. Data are presented as the mean \pm standard deviation of 10 independent experiments. Values are compared between resting and LPS, +YVAD, $+\mathrm{Nec}-1$ or $+\mathrm{Nec}-1 \mathrm{i}$. ${ }^{*} \mathrm{P}<0.05,{ }^{* *} \mathrm{P}<0.01$. LPS, lipopolysaccharide; IL-1 $\beta$, interleukin-1 $\beta$; Nec-1, Necrostatin-1.

adaptor kinase RIP1, inhibits necroptosis (19). YVAD did not affect the release of HMGN1 or LDH from LPS-stimulated RAW264.7 cells (Figs. 1 and 2), although it inhibited the release of IL-1 $\beta$ as a marker of caspase-1 activation (Fig. 3). Similarly, Nec-1 did not affect HMGN1 release or LDH release from LPS-stimulated RAW264.7 cells (Figs. 1 and 2). A previous study confirmed that Nec-1 suppressed the release of HMGN1 from RAW264.7 cells treated with LPS plus zVAD (a pan-caspase inhibitor), which induces necroptosis of macrophages $(20,21)$.

These observations suggest that HMGN1 release from LPS-stimulated RAW264.7 cells is not associated with pyroptosis (caspase-1-acivated cell death) or necroptosis (RIP1-dependent programmed cell death).

Involvement of necrosis in HMGN1 release from LPS-stimulated RAW264.7 cells. Finally, the involvement 
A

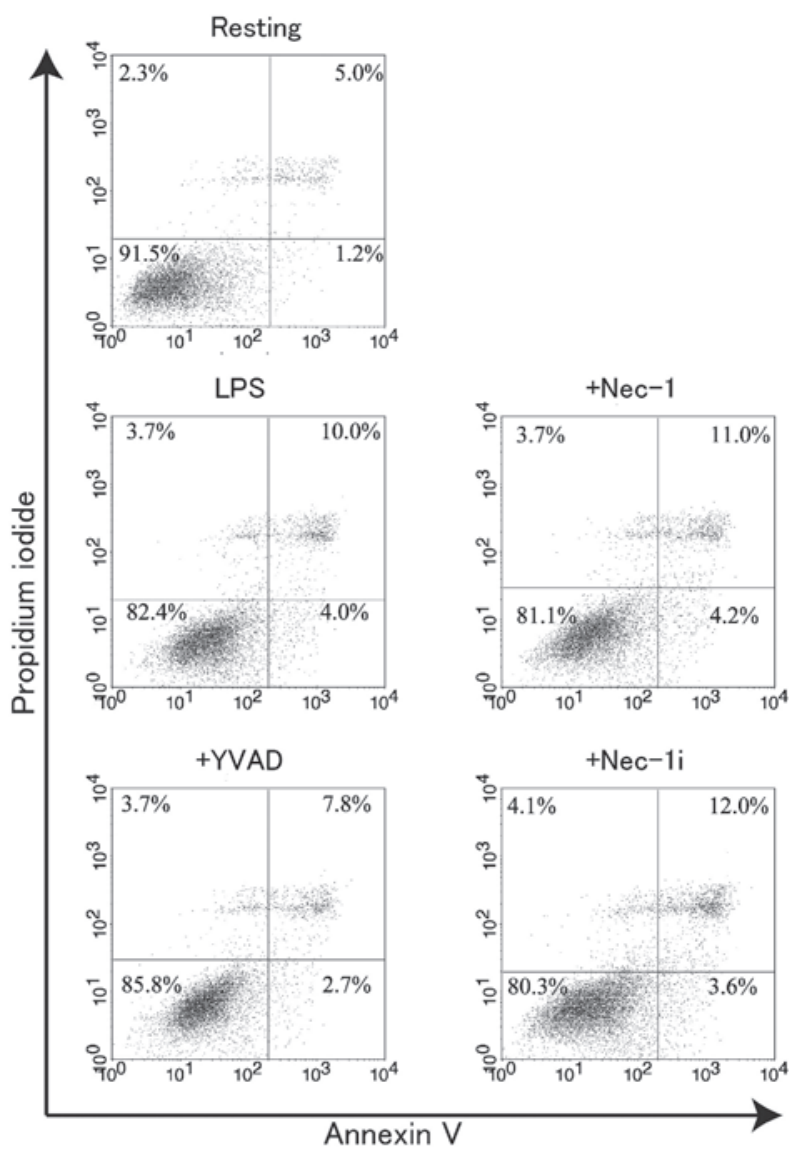

B

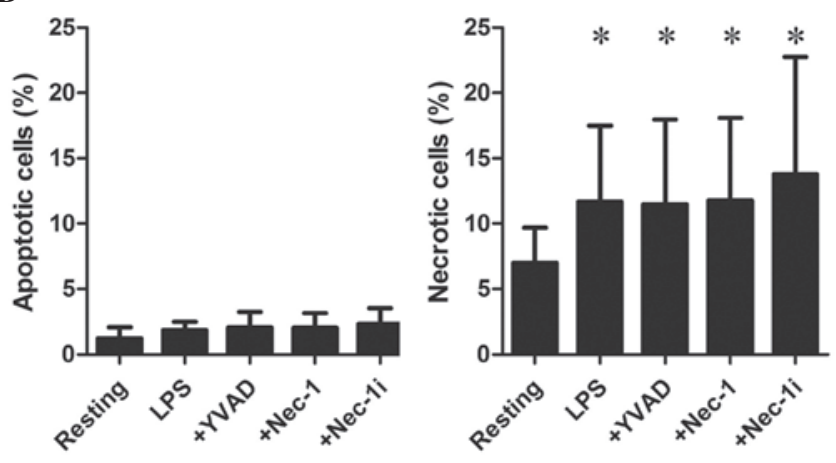

Figure 4. Assay for apoptosis and necrosis of LPS-stimulated RAW264.7 cells. RAW264.7 cells were incubated with LPS $(100 \mathrm{ng} / \mathrm{ml})$ or without (resting) in the absence or presence of YVAD, Nec-1 or Nec-1i (10 $\mu \mathrm{M}$ each) at $37^{\circ} \mathrm{C}$ for $8 \mathrm{~h}$, and then stained with fluorescein isothiocyanate-labeled annexin $\mathrm{V}$ and PI. (A) Representative plots showing the distribution of annexin V and PI staining for resting and LPS-stimulated cells incubated with or without YVAD, Nec-1 or Nec-1i. The percentage of the cells delimited by each quadrant is also shown. Images are representative of 10 separate experiments. (B) Quantitative analysis of the percentage of apoptotic and necrotic cells. Apoptotic and necrotic cells were defined as annexin $\mathrm{V}^{+} / \mathrm{PI}^{-}$(lower right) and annexin $\mathrm{V}^{+} / / \mathrm{PI}^{+}$(upper left + right), respectively. Data are presented as the mean \pm standard deviation of 10 independent experiments. Values are compared between resting and LPS, +YVAD, $+\mathrm{Nec}-1$ or $+\mathrm{Nec}-1 \mathrm{i}$. ${ }^{*} \mathrm{P}<0.05$. LPS, lipopolysaccharide; PI, propidium iodide; Nec-1, Necrostatin-1.

of necrotic cell death in HMGN1 release was determined by staining LPS-stimulated RAW264.7 cells with annexin V and PI. As shown in Fig. 4A and B, LPS stimulation did not significantly increase apoptotic cells (lower right division) but significantly increased necrotic cells $(\mathrm{P}<0.05$, upper right and left divisions) compared with resting. Of note, it has been reported that PI-positive cells are increased by pyroptosis and necroptosis $(22,23)$. However, YVAD and Nec-1 did not affect these patterns of cell death. Together these findings suggest that LPS stimulation is not likely to induce apoptosis, pyroptosis or necroptosis of RAW264.7 cells, and HMGN1 is released from PI-positive necrotic RAW264.7 cells stimulated with LPS.

\section{Discussion}

Endotoxin/septic shock is a severe and abnormal condition that is induced during infections with gram-negative bacteria. It is characterized by systemic inflammatory responses of the host to invading microorganisms, PAMPs and damage-associated molecular patterns, including endogenous danger signal molecules termed alarmins (4). A previous study revealed that HMGN1, an alarmin, acts as a cytokine in the extracellular milieu, which enhances Th1-type antigen-specific immune responses (13). In addition, HMGN1 stimulates immune cells, including monocytes and dendritic cells, to secrete inflammatory cytokines via the TLR4/NF- $\kappa \mathrm{B}$ and MAPK pathways (14). Since the activation of NF- $\kappa B$ and excessive production of inflammatory cytokines from monocytes/macrophages are key events in sepsis, the extracellular release of HMGN1 from monocytes/macrophages is expected to be important in the pathogenesis of sepsis/septic shock (14). However, the release mechanism of HMGN1 from monocytes/macrophages remains to be elucidated. The present study revealed that HMGN1 is extracellularly released from LPS-stimulated RAW264.7 macrophage-like cells, accompanied by unprogrammed necrotic cell death but not pyroptosis, necroptosis or apoptosis.

Cell death has been discussed dichotomously as either apoptosis or necrosis (24). Apoptosis is typically described as a programmed process of self-destruction that avoids release of inflammatory cellular contents (25). The execution of apoptosis minimizes the leakage of cellular constituents by forming an apoptotic body, a result of non-lytic cellular shrinkage (26). By contrast, necrosis is the most common form of cell death, which is characterized by membrane rupture and leakage of prophlogistic cellular constituents (24). Thus, necrosis is considered to be the potent source of immunoactivating danger signal molecules, including alarmins. In addition, a previous study expanded categorization of the two types of novel cell deaths i.e. pyroptosis and necroptosis (26). Pyroptosis is identified as caspase-1-dependent cell death of macrophages and dendritic cells found in bacterial infection (27). Pyroptosis is induced by the formation of the inflammasome that facilitates the activation of caspase-1, and the generation of cytokines, including IL-1 $\beta$, based on the cleavage of their precursors by activated caspase-1 (12). During pyroptosis, cellular contents are rapidly released into the extracellular space by pore formation and plasma membrane loss (28). Another form of cell death, necroptosis was originally defined as a potent immunogenic programmed cell death induced by the presence of zVAD-fmk (a pan-caspase inhibitor) and tumor necrosis factor receptor signaling that involves the activation of RIP1 and mitochondrial instability $(20,29)$. During necroptosis, swelling of cellular organelles and plasma membrane disruption are induced, and consequently inflammatory cellular contents are extracellularly released (25). 
In the present study, it was revealed that HMGN1 is released from LPS-stimulated RAW264.7 cells, accompanied by cell death as assessed by the release of LDH. Subsequently, the patterns of cell death involved in HMGN1 release from LPS-stimulated RAW264.7 cells were determined using YVAD (a caspase-1 inhibitor) and Nec-1 (a RIP1 inhibitor). YVAD and Nec-1 did not alter LPS-induced HMGN1 and $\mathrm{LDH}$ release, although YVAD and Nec-1 inhibited LPS-stimulated IL-1 $\beta$ release and LPS/zVAD-stimulated HMGN1 release, respectively. These observations suggest that pyroptosis (caspase-1-activated cell death) and necroptosis (RIP1-dependent programmed cell death) are not involved in the release of HMGN1 from LPS-stimulated RAW264.7 cells. In addition, flow cytometric analysis indicated that LPS stimulation did not induce apoptosis but substantially augmented necrosis, as evidenced by annexin V/PI-staining. Together these findings suggest that HMGN1 is released from necrotic (unprogrammed cell death) but not apoptotic, pyroptotic or necroptotic (a programmed form of necrosis) RAW264.7 cells following stimulation with LPS. Supporting this theory, pyroptosis and necroptosis were not induced in RAW264.7 cells by LPS stimulation under our experimental condition, since YVAD (a pyroptosis inhibitor) and Nec-1 (a necroptosis inhibitor) did not inhibit LPS-induced LDH release and PI staining, although these inhibitors were capable of suppressing LDH release and PI staining associated with pyroptosis and necroptosis (27,30-32). In addition, IL-1 $\beta$ was extracellularly released from RAW264.7 cells by LPS stimulation, but its maximum level was $30 \mathrm{pg} / \mathrm{ml}$ (Fig. 3). Notably, pyroptosis-associated IL-1 $\beta$ release is reported to reach $1-2 \mathrm{ng} / \mathrm{ml}(11,33,34)$. These observations suggest that in our experiment, caspase-1 is activated by LPS stimulation to process and release IL-1 $\beta$; however, it is unlikely that the activation is sufficient to induce pyroptosis.

In conclusion, the release mechanism of HMGN1 from macrophages was evaluated using RAW264.7 cells and it was revealed that its extracellular release from LPS-stimulated RAW264.7 cells is predominantly dependent on necrosis (unprogrammed cell death) but not apoptosis, pyroptosis or necroptosis. Under conditions of sepsis/septic shock, alarmins are hypothesized to be released from immune cells due to cell death or cell activation at the site of infection and inflammation, and have a fundamental role in the regulation of host defense and tissue repair $(35,36)$. Therefore, HMGN1 released from macrophages and other immune cells by stimulation with PAMPs, including LPS, is likely to be important in sepsis/septic shock as an alarmin. Anti-cytokine based therapies for sepsis-associated systemic inflammation have not been successful (37). Therefore, the modulation of cell death/cell activation and subsequent release of alarmins, including HMGN1 is expected to be a promising target for a novel therapeutic strategy against sepsis/septic shock.

\section{Acknowledgements}

The present study was supported in part by a Grant-in-Aid for Young Scientists B and for Scientific Research C (grant no. 2290416) from the Japan Society for the Promotion of Science, and a Grants-in-Aid (grant no. S1201013) from the Ministry of Education, Culture, Sports, Science and Technology (Japan).

\section{References}

1. Cohen J: The immunopathogenesis of sepsis. Nature 420: 885-891, 2002

2. O'Brien JM Jr, Ali NA, Aberegg SK and Abraham E: Sepsis. Am J Med 120: 1012-1022, 2007.

3. Van Amersfoort ES, Van Berkel TJ and Kuiper J: Receptors, mediators, and mechanisms involved in bacterial sepsis and septic shock. Clin Microbiol Rev 16: 379-414, 2003.

4. Manson J, Thiemermann C and Brohi K: Trauma alarmins as activators of damage-induced inflammation. Br J Surg 99 (Suppl 1): S12-S20, 2012.

5. Tewary P, de la Rosa G, Sharma N, Rodriguez LG, Tarasov SG, Howard OM, Shirota H, Steinhagen F, Klinman DM, Yang D and Oppenheim JJ: $\beta$-Defensin 2 and 3 promote the uptake of self or CpG DNA, enhance IFN- $\alpha$ production by human plasmacytoid dendritic cells, and promote inflammation. J Immunol 191: 865-874, 2013.

6. Nagaoka I, Tamura $\mathrm{H}$ and Hirata $\mathrm{M}$ : An antimicrobial cathelicidin peptide, human CAP18/LL-37, suppresses neutrophil apoptosis via the activation of formyl-peptide receptor-like 1 and P2x7. J Immunol 176: 3044-3052, 2006.

7. Suzuki K, Murakami T, Kuwahara-Arai K, Tamura H, Hiramatsu K and Nagaoka I: Human anti-microbial cathelicidin peptide LL-37 suppresses the LPS-induced apoptosis of endothelial cells. Int Immunol 23: 185-193, 2011.

8. Moussion C, Ortega N and Girard JP: The IL-1-like cytokine IL-33 is constitutively expressed in the nucleus of endothelial cells and epithelial cells in vivo: A novel 'alarmin'? PLoS One 3: e3331, 2008.

9. Bianchi ME: DAMPs, PAMPs and alarmins: All we need to know about danger. J Leukoc Biol 81: 1-5, 2007.

10. Yang Z, Li L, Chen L, Yuan W, Dong L, Zhang Y, Wu H and Wang C: PARP-1 mediates LPS-induced HMGB1 release by macrophages through regulation of HMGB1 acetylation. J Immunol 193: 6114-6123, 2014.

11. Hu Z, Murakami T, Suzuki K, Tamura H, Kuwahara-Arai K, Iba T and Nagaoka I: Antimicrobial cathelicidin peptide LL-37 inhibits the LPS/ATP-induced pyroptosis of macrophages by dual mechanism. PLoS One 9: e85765, 2014.

12. Lamkanfi M and Dixit VM: Modulation of inflammasome pathways by bacterial and viral pathogens. J Immunol 187: 597-602, 2011.

13. Wei F, Yang D, Tewary P, Li Y, Li S, Chen X, Howard OM, Bustin M and Oppenheim JJ: The Alarmin HMGN1 contributes to antitumor immunity and is a potent immunoadjuvant. Cancer Res 74: 5989-5998, 2014.

14. Yang D, Postnikov YV, Li Y, Tewary P, de la Rosa G, Wei F, Klinman D, Gioannini T, Weiss JP, Furusawa T, et al: High-mobility group nucleosome-binding protein 1 acts as an alarmin and is critical for lipopolysaccharide-induced immune responses. J Exp Med 209: 157-171, 2012.

15. Magna M and Pisetsky DS: The role of HMGB1 in the pathogenesis of inflammatory and autoimmune diseases. Mol Med 20: 138-146, 2014.

16. Murakami T, Suzuki K, Tamura H and Nagaoka I: Suppressive action of resolvin D1 on the production and release of septic mediators in D-galactosamine-sensitized endotoxin shock mice. Exp Ther Med 2: 57-61, 2011.

17. Kugler JE, Horsch M, Huang D, Furusawa T, Rochman M, Garrett L, Becker L, Bohla A, Hölter SM, Prehn C, et al: High mobility group $\mathrm{N}$ proteins modulate the fidelity of the cellular transcriptional profile in a tissue- and variant-specific manner. J Biol Chem 288: 16690-16703, 2013.

18. Gerlitz G: HMGNs, DNA repair and cancer. Biochim Biophys Acta 1799: 80-85, 2010.

19. Cho Y, McQuade T, Zhang H, Zhang J and Chan FK: RIP1-dependent and independent effects of necrostatin-1 in necrosis and T cell activation. PLoS One 6: e23209, 2011.

20. Kaczmarek A, Vandenabeele P and Krysko DV: Necroptosis: The release of damage-associated molecular patterns and its physiological relevance. Immunity 38: 209-223, 2013.

21. Moquin DM, McQuade T and Chan FK: CYLD deubiquitinates RIP1 in the TNF $\alpha$-induced necrosome to facilitate kinase activation and programmed necrosis. PLoS One 8: e76841, 2013. 
22. Wree A, Eguchi A, McGeough MD, Pena CA, Johnson CD, Canbay A, Hoffman HM and Feldstein AE: NLRP3 inflammasome activation results in hepatocyte pyroptosis, liver inflammation and fibrosis in mice. Hepatology 59: 898-910, 2014.

23. Khan MJ, Rizwan Alam M, Waldeck-Weiermair M, Karsten F, Groschner L, Riederer M, Hallström S, Rockenfeller P, Konya V, Heinemann A, et al: Inhibition of autophagy rescues palmitic acid-induced necroptosis of endothelial cells. J Biol Chem 287: 21110-21120, 2012.

24. Zong WX and Thompson CB: Necrotic death as a cell fate. Genes Dev 20: 1-15, 2006.

25. Davidovich P, Kearney CJ and Martin SJ: Inflammatory outcomes of apoptosis, necrosis and necroptosis. Biol Chem 395: 1163-1171, 2014.

26. Inoue $\mathrm{H}$ and Tani $\mathrm{K}$ : Multimodal immunogenic cancer cell death as a consequence of anticancer cytotoxic treatments. Cell Death Differ 21: 39-49, 2014.

27. Cervantes J, Nagata T, Uchijima M, Shibata K and Koide Y: Intracytosolic listeria monocytogenes induces cell death through caspase-1 activation in murine macrophages. Cell Microbiol 10: 41-52, 2008.

28. Lamkanfi $M$ and Dixit VM: Mechanisms and functions of inflammasomes. Cell 157: 1013-1022, 2014.

29. Degterev A, Huang Z, Boyce M, Li Y, Jagtap P, Mizushima N Cuny GD, Mitchison TJ, Moskowitz MA and Yuan J: Chemical inhibitor of nonapoptotic cell death with therapeutic potential for ischemic brain injury. Nat Chem Biol 1: 112-119, 2005.
30. YamanakaK,Urano Y,Takabe W,Saito Y andNoguchiN: Induction of apoptosis and necroptosis by 24(S)-hydroxycholesterol is dependent on activity of acyl-CoA: Cholesterol acyltransferase 1. Cell Death Dis 5: e990, 2014.

31. Steinhart L, Belz K and Fulda S: Smac mimetic and demethylating agents synergistically trigger cell death in acute myeloid leukemia cells and overcome apoptosis resistance by inducing necroptosis. Cell Death Dis 4: e802, 2013.

32. Tseng WA, Thein T, Kinnunen K, Lashkari K, Gregory MS, D'Amore PA and Ksander BR: NLRP3 inflammasome activation in retinal pigment epithelial cells by lysosomal destabilization: Implications for age-related macular degeneration. Invest Ophthalmol Vis Sci 54: 110-120, 2013.

33. Sauer JD, Witte CE, Zemansky J, Hanson B, Lauer P and Portnoy DA: Listeria monocytogenes triggers AIM2-mediated pyroptosis upon infrequent bacteriolysis in the macrophage cytosol. Cell Host Microbe 7: 412-419, 2010.

34. Hua KF, Chou JC, Ka SM, Tasi YL, Chen A, Wu SH, Chiu HW, Wong WT, Wang YF, Tsai CL, et al: Cyclooxygenase-2 regulates NLRP3 inflammasome-derived IL-1 $\beta$ production. J Cell Physiol 230: 863-874, 2015.

35. Diener KR, Al-Dasooqi N, Lousberg EL and Hayball JD: The multifunctional alarmin HMGB1 with roles in the pathophysiology of sepsis and cancer. Immunol Cell Biol 91: 443-450, 2013.

36. Wang H, Ward MF and Sama AE: Targeting HMGB1 in the treatment of sepsis. Expert Opin Ther Targets 18: 257-268, 2014

37. Webster NR and Galley HF: Immunomodulation in the critically ill. Br J Anaesth 103: 70-81, 2009. 